\title{
Advance Evaluation of System Protection Control Strategy Based on Multi-scheme Concurrency
}

\author{
Lin Liu ${ }^{1}$, Jian $\mathrm{Guo}^{2}$, Jianyu $\mathrm{Luo}^{1}$, Xiongfeng $\mathrm{Xu}^{2}$, Kaiming Luo ${ }^{1}$, and Shengming Wang ${ }^{2}$ \\ ${ }^{1}$ State Grid Jiangsu Electric Power Co., Ltd., Nanjing 210024, Jiangsu, China \\ ${ }^{2}$ NARI Group Corporation / State Grid Electric Power Research Institute, Nanjing 211106, Jiangsu, China
}

\begin{abstract}
According to rapid assessment requirement of system protection control strategy adaptability in future operation mode, combining with application status of distributed computing for power system security and stability analysis, based on design idea of asynchronism and concurrency, this paper proposes a multischeme concurrent computing framework suitable for coarse-grained case parallelism. By the way of multidata section parallel, the asynchronous and concurrent scheduling management of computing task of multiple application functions under different sections is realized, which effectively improves the utilization rate of computing resources and significantly reduces the computing period of advance evaluation of system protection control strategy adaptability. The technology has been applied in provincial power grid and its effectiveness has been verified.
\end{abstract}

\section{Introduction}

Due to the disadvantages of traditional safety prevention and control, such as single control measure, lack of overall consideration, lack of coordination control and difficulty in effectively dealing with cascading faults. In response to serious faults such as UHV DC blocking, the safe operation of power grid is faced with great risks [1]. In order to shorten the response time of UHV DC fault disposal, improve accident handling efficiency and ensure the safe operation of large power grids, State Grid Corporation has built a system protection and accurate load cutting system [2-4]. In order to meet the rapid assessment requirement of the system protection control strategy adaptability, it is necessary to further improve the speed of parallel computation of security and stability analysis, the flexibility of calculation process definition and the extensibility of application functions.

The existing parallel computing framework that based on single data section mainly has problems as follows: (1) it takes too long to serially calculate the power flow of multiple data sections on the application server costs. It is necessary to encapsulate the function that forms the power flow data of future operation mode and perform it in parallel on the computer cluster to shorten the data integration time [5-6]; (2) it organizes the computing procedure of only one data section at a time by the computer cluster. As task decomposition and results integration at the node of data download and upload as well as management, the computer cluster is in idle and waiting state [7]. For multiple section data, it is required to further optimize the interaction mechanism between the application server and the computer cluster as well as the computing management mechanism inside the computer cluster, so as to reduce the idle and waiting time of the computer cluster and further improve the computing efficiency of the computer cluster.

At present, the following three parallel computing technologies are mainly adopted to improve the overall computing speed: (1) Improve the computing speed of all computing tasks of the whole function through the case parallel scheme [8-9]; (2) Improve the computing speed of a single case through the algorithm parallel scheme [1011]. (3) Improve the computing speed of static security analysis through multi-period parallel mechanism [12]. Paper [13] improves the computing speed of static security checking based on parallel computing and data reuse. Paper [14] realizes a rapid assessment of the change trend of the future operation mode of the power grid. Paper [15] proposes a distributed parallel computing management scheme based on time, space and application dimensions for state estimation, dispatcher power flow and other applications. These research results improve the computing speed from the aspects of multi-data section of a single application function or multi-application function computing tasks of a single data section. However, for static stability assessment and transient stability assessment including multiple time intervals, the overall computing speed cannot be improved only by the performance improvement of a single application function and the performance optimization of a single section.

In order to meet the requirement of rapid assessment of system protection control strategy adaptability in future operation mode, based on the design idea of asynchronism and concurrency, this paper proposes a multi-scheme concurrent computing framework suitable for coarsegrained case parallelism. Asynchronous and concurrent

xuxiongfeng@sgepri.sgcc.com.cn 
scheduling management of computing tasks of multiple application functions under different sections are realized through multi-data sections in parallel, which effectively improve the utilization of computing resources. The computing period of advance evaluation of system protection control strategy adaptability is reduced significantly under the condition of constant computing resources.

\section{General framework}

The multi-scheme concurrent computing framework suitable for coarse-grained case parallelism meets the requirements of multi-data section and multi-application concurrent computing, it has the following advantages: (1) The computing procedure is configurable. It can dynamically select the corresponding execution branch according to the computing results of relevant application functions, making the computing procedure configuration more flexible. (2) Making full use of computing resources. Through asynchronous concurrent management, idle computing resources can also be utilized when downloading computing data, uploading computing results, decomposing tasks performed by management nodes and aggregating results.

The multi-scheme concurrent computing hardware consists of application server and computer cluster. The application server realizes the unified interaction of model and data as well as the integrated display of humanmachine interface. The computer cluster mainly realizes the data integration of future operation mode and the parallel processing of the static and transient security and stability assessment taking into account system protection control strategy. The framework for concurrent computing is shown in Figure1. To make full use of the computing power of the computer cluster, the parallel processing of different data sections is realized by running multiple instances of data integration process of future operation mode on the computer cluster, so as to reduce the generation time of multiple power flow data section of future operation mode. After data integration, security and stability assessment is concurrently carried out on the computer cluster. By means of multi-scheme concurrency mechanism, the management node supports the simultaneous operation of security assessment computing tasks of multiple data sections. The computing power of computing nodes is efficiently used, thus reducing the computing period of advance evaluation of system protection control strategy.

In general, multi-scheme concurrent computing software is divided into two modules: advance evaluation parallel management and multi-scheme concurrent computing management. In which, the advance evaluation parallel management module runs on the application server, receives the advance evaluation request of system protection control strategy, controls the operation process of the subsequent integration of multiple data sections and safety and stability assessment, and is in charge of the scheduling management of computing data of multiple periods under the computer cluster multi-scheme. The multi-scheme concurrent computing management module runs on the computer cluster, supports running multiple independent schemes on management nodes simultaneously. Each scheme independently conducts computing procedure management, interacts with the advance evaluation parallel management module on the application server, receives the issued computing data, runs the program associated with relevant application functions according to the startup conditions, and finally, returns the computing results to the advance evaluation parallel management module.

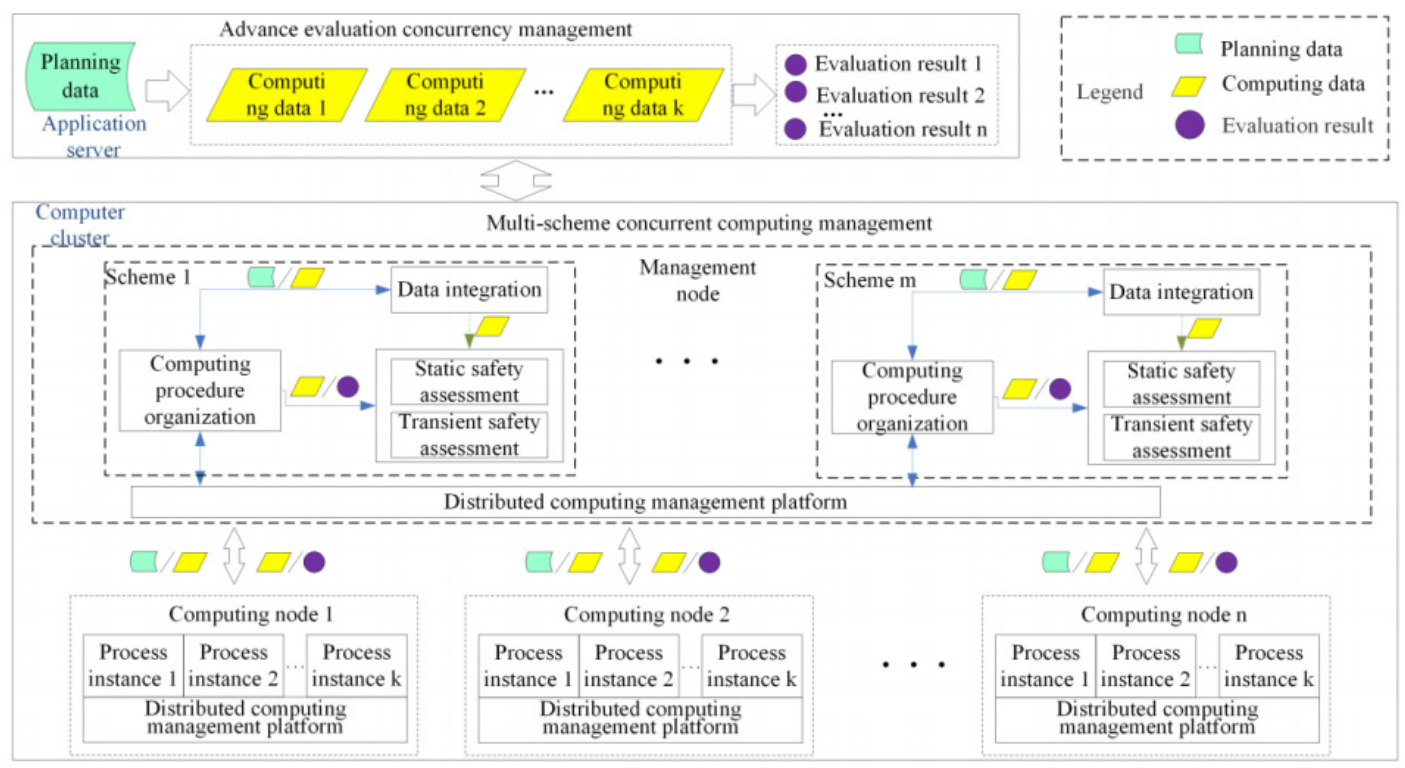

Figure 1. Multi-scheme concurrent computing framework. 


\section{Key technology}

\subsection{Configuration of computing procedure}

In order to improve the flexibility and adaptability of the calculation process definition, based on the design idea of workflow [16-18], the application functions of the advance evaluation of the system protection control strategy are unified encapsulated into a process. Taking the data related to each application function as transfer information (including startup conditions), the various application functions of security and stability analysis and computing are organized in a standardized and normalized way. The computing procedure organization engine analyzes the pre-defined security and stability analysis operation flow. Then, according to the actual computing state of each application function, select the corresponding execution branch and run the corresponding application function procedure, as shown in Figure 2.

The computing procedure definition consists of the computing function zone, the computing task (or the computing task group), the start condition and other keywords, as well as operators such as various operands, arithmetic operators and logical operators. The computing procedure of security assessment is composed of several computing function zones, and is executed serially by each computing function zone. A number of computing tasks (or groups) can be defined in each computing function zone. A computing task corresponds to an application function, multiple computing tasks can form a group of computing tasks. Each (or group) computing task can define a separate startup condition, which consists of operands such as keywords, variables, constants, and various arithmetic operators and logical operators. After a computing task (application function) is completed, the output of some data item variables are supported as the input variable of startup condition for other application function. All computing tasks in each computing function zone concurrently conduct startup condition judgment, and the computing task will be executed immediately after startup condition is set up.

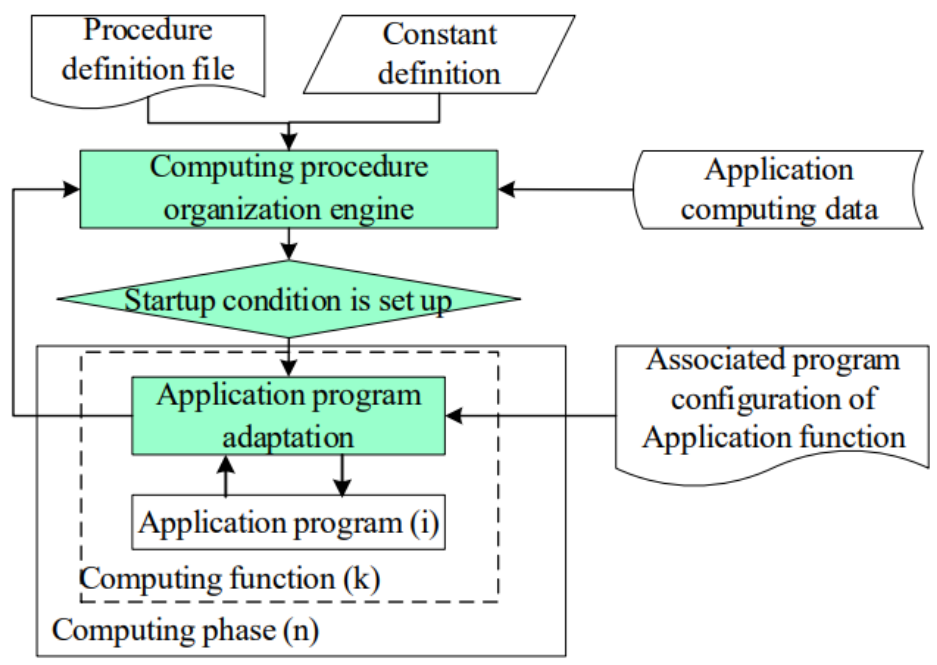

Figure 2. Flow chart of computing procedure analysis and application function integration.

The specific implementation adopts a similar expression to the scripting language, and achieves a unified description of the computing procedure of security and stability analysis by defining relevant keywords and variables. Specific keywords are shown in Table 1.

Table 1. Keywords of computing procedure definition.

\begin{tabular}{ll}
\hline Keywords & Explanation of meaning \\
\hline [Computing phase starts] & Start mark of computing phase definition \\
[Computing phase ends] & End mark of computing phase definition \\
var & var(): Defined variable values in () \\
taskgroup & Definition mark of computing task set \\
task & Definition mark of computing task \\
sta & sta (): Defined variable state values in () \\
val & val (): Directly defined values in () \\
if & Start mark of definition condition \\
then & End mark of branch 1 definition condition, start mark of branch 1 (The next row \\
else & is branch 1) \\
end if & Start mark of branch 2 (The next row is branch 2), optional item \\
\hline
\end{tabular}




\subsection{Asynchronous concurrent management}

The working domain management mechanism of the existing concurrent computing framework has realized the autonomy of computing procedure management, at the same time, it manages the computing resources according to three levels: working domain - computing node computing procedure. On the one hand, it can avoid the performance bottleneck of centralized scheduling, on the other hand, it can also prevent the application functions of formulating computing schemes based on available computing resources from taking up too much computing resources [19-21]. However, the computing procedure of only one data section can be organized at a time in a work domain, and there are idle computing resources during the data collection, data uploading and downloading at the management node.

In order to improve the utilization efficiency of computing resources and avoid idle waiting time caused by synchronization mechanism in computing task scheduling and computing result recovery, in parallel computing management platform, asynchronous concurrent management mechanism is adopted to realize the scheduling management of computing tasks. Generally speaking, it can be divided into two levels: operation mode and application function. Through asynchronous concurrent management mechanism, the computing power of the computing node can be effectively utilized when the management node is processing relevant data. Each operation mode runs asynchronously and concurrently on the management node to realize the asynchronous operation of computing data download, computing procedure management and computing result upload, so as to avoid the idle waiting state of computing node when the management node in a single operation mode is downloading computing data or uploading computing result. In order to reduce the additional data transmission and management overhead of application functions during computing task scheduling, the distributed computing management platform supports to determine the optimal scheduling granularity of each application function according to the number of computing tasks and the estimated execution time of each application function [22]. While making full use of computing resources, the scheduling round is reduced as far as possible to shorten the time of platform scheduling and management.

\section{Analysis of examples}

The distributed computing management platform based on multi-scheme concurrent computing framework has been applied to the whole process intelligent analysis and control decision system of system protection action in a provincial power grid which contains about 4,000 nodes. The system hardware consists of two application servers. The computer cluster consists of six computing nodes. The management node runs concurrently in four schemes mode, and each computing node runs 16 computing procedure instances. The system protection control strategy advance evaluation system includes data integration, static safety analysis, transient stability analysis and emergency state pre-decision function. The emergency state pre-decision only runs when the safety assessment result is unsafe. The acceleration ratio of advance evaluation is defined as the ratio of the computing time of the data sections of four future methods (including data integration) under the single scheme mode of concurrency and the computing time under the four schemes operation mode.

In the single scheme mode, the theoretical computing time $T_{1}$ of the four data sections completing the advance evaluation is shown in equation (1).

$$
T_{1}=4 \times\left(t_{\mathrm{dd}}+t_{\mathrm{fs}}+t_{\mathrm{c}}+t_{\mathrm{fn}}+t_{\mathrm{rp}}\right)
$$

Where, $t_{d d}, t_{f s}, t_{f n}$ and $t_{r p}$ are respectively the time consumed by the management node data download, management node application function computing task generation, management node result recovery and summary, and management node result upload module. $t_{c}$ is the average time to complete all security and stability assessment tasks for a single data section.

In practical multi-scheme mode, the execution time for a single data section to complete all the security and stability assessment computing tasks on the computer cluster generally exceeds the management overhead time of the distributed computing management platform, that is $t_{c c} \geq t_{d d}+t_{f s}+t_{f n}+t_{r p}$. In the multi-scheme mode of concurrent management node, one scheme management overhead of the distributed computing management platform can be completed in parallel during the execution of other computing tasks. Therefore, the theoretical fastest time $T_{2}$ for the four data sections to complete the safety and stability assessment is shown in equation (2).

$$
T_{2}=t_{\mathrm{dd}}+t_{\mathrm{fs}}+4 \times t_{\mathrm{c}}{ }^{\prime}+t_{\mathrm{fn}}+t_{\mathrm{rp}}
$$

Where, $t_{c}{ }^{\prime}$ is the average time to complete all the security and stability assessment tasks of a single data section in multi-scheme concurrent mode. In multischeme concurrent mode, due to the computing tasks of multiple data sections are running simultaneously, the synchronization wait time of computing tasks on computing nodes is reduced, therefore, the average execution time of single data section safety and stability assessment in multi-scheme concurrent mode is generally lower than that in single-scheme operation mode, $t_{c}{ }^{\prime} \leq t_{c}$. As a result, the saved time for security and stability assessment in multi-scheme concurrent mode compared with single-scheme operation mode $\Delta T_{\mathrm{s}}$ is shown in equation (3).

$$
\Delta T_{s}=T_{1}-T_{2} \approx(4-1) \times\left(t_{d d}+t_{f s}+t_{f n}+t_{r p}\right)
$$

Four cases of actual data sections are selected and the specific statistical results are shown in Table 2. As can be seen from Table 2, in the single scheme mode, due to the data integration serial processing and the idle computing resources, the final four data sections take 724 seconds. After it is changed to the four schemes mode concurrent mode, the data integration is also processed in parallel and makes full use of computing resources. The average speed-up ratio of the data section safety and stability assessment (data integration, static security analysis and transient stability analysis) in the four periods reaches 1.33 , 
and the computing time is reduced to 547 seconds. The performance is satisfied in advance evaluation. computing time is shortened and the computing

Table 2. Statistics results of advance evaluation with single scheme and four schemes.

\begin{tabular}{ccccc}
\hline Test case & $\begin{array}{c}\text { Number of } \\
\text { contingency faults }\end{array}$ & $\begin{array}{c}\text { Computing time of } \\
\text { single scheme / s }\end{array}$ & $\begin{array}{c}\text { Computing time of four } \\
\text { schemes / s }\end{array}$ & Speed-up ratio \\
\hline Case 1 & 496 & $4 * 181$ & 557 & 1.30 \\
Case 2 & 496 & $4 * 181$ & 536 & 1.35 \\
Case 3 & 496 & $4 * 181$ & 524 & 1.38 \\
Case 4 & 496 & $4 * 181$ & 570 & 1.27 \\
Average & 496 & 724 & 547 & 1.33 \\
\hline
\end{tabular}

\section{Conclusion}

In this paper, a multi-scheme concurrent computing framework adapted to coarse-grained parallelism is proposed. On the premise of keeping existing computing resources and core algorithm logic of application functions unchanged, based on the multi-scheme concurrency mechanism, the control strategy assessment task of multi-period data section is implemented asynchronously and concurrently on the computer cluster. It can improve the utilization efficiency of computing resources, effectively shorten the computing time of stability analysis, and meet the requirement of computing time for advance evaluation of system protection control strategy. In the next step, it is necessary to carry out research on related distributed computing performance optimization technology to further reduce computing time under the condition of scalable computing resource scale.

\section{Acknowledgements}

This work was supported by State Grid Jiangsu Electric Power Co., Ltd. (The Science and Technology Project of "Research on intelligent analysis and control decisionmaking technology in the whole process of system protection action", Contract No.: SGJS0000DKJS1900572).

\section{References}

1. Chen Guoping. (2018) Guest Chief Editor's Note. J. Automation of Electric Power Systems., 42 (22):1.

2. Mohammad F. (2020) Ultra-high-speed non-unit nondifferential protection scheme for buses of MMCHVDC grids. J. IET Renewable Power Generation.,14(9): 1541-1549.

3. Li Botong, Li Chunbo, Li Bin, Wen Weijie. (2020) Study on the distributed-parameter resistance earth model and potential distribution of the monopoleground-return HVDC. J. Electric Power Systems Research., 187.

4. Chen Qing, Shan Xin, Luo Jianyu. (2017) Sourcegrid-load Coordinated Control Strategy and Its Application Under UHVDC Faults. J. Automation of Electric Power Systems., 41(5):147-152.

5. Fang Yongjie, Wang Shengming, Xu Tasihan. (2018)
Concurrent computing architecture for power system security stability analysis based on process configuration. J. Power System Protection and Control., 46(11): 92-98.

6. Bo Zhiqian, Lin Xiangning, Wang Qingping. (2016) Developments of power system protection and control. J. Protection and Control of Modern Power Systems., 1(1): 1-8.

7. Wang Shengming, $\mathrm{Xu}$ Tasishan, Guo jian. (2017) Multi-Scheme Concurrent Security Check of Dispatching Schedule. J. Southern Power System Technology., 11(5): 56-62.

8. Li Bijun, $\mathrm{Xu}$ Jianbing, $\mathrm{Xu}$ Taishan. (2008) Engineering application of integrated and coordinated defense technology of large power system security and stability. J. Automation of Electric Power Systems., 32(6): 25-30.

9. Yan Jianfeng, Yu Zhihong, Tian Fang. (2008) Dynamic security assessment \& early warning system of power system. J. Proceedings of the CSEE., 28(34): 87-93.

10. Jiang Han, Jiang Quanyuan. (2013) A parallel transient stability algorithm for large-scale power system based on GPU platform. J. Power System Protection and Control., 41(4): 13-20.

11. Wang Xingzhi, Yan Zheng, Shen Chen. (2011) Grid computing of operation scheduling with security constraint based on SOA. J. Power System Protection and Control., 39(24): 90-95.

12. Liu Gaoming, Song Wei, Qiu Xiangdong. (2014) A P2P based hybrid parallel algorithm for on-line checking protection setting. J. Southern Power System Technology., 8(2): 60-64.

13. Li Feng, Li Hucheng, Yu Yijun. (2013) Fast computing technologies for static security checking based on parallel computation and data reuse. J. Automation of Electric Power Systems., 37(14): 7580.

14. Yan Jianfeng, Feng Changyou, Lu Guangming. (2015) On-line trend analysis technology of large power grid considering operation mode arrangement. J. Automation of Electric Power Sytems39-(1), 111-116.

15. Zhang Haibo, Zhu Cunhao. (2016) Distributed parallel calculation management mechanism considering time-space and application dimensions. J. Automation of Electric Power Systems., 40(15): 106112. 
16. Xu Liang, Zhang Li, Fan Zhiqiang. (2010) An approach of real-time work-flow modeling based on UML. J. Journal of Computer Research and Development., 47(7): 1184-1191.

17. H.Tabatabaee. (2013) Work Flow Scheduling Modeling in Grid Computing Using Linear Switching State Space. In: International Scientific Academy of Engineering and Technology. Dubai, UAE. pp. 80-85.

18. Sun Weizhen, Wang He. (2019) Enhancing flexibility method of monitoring system based on workflow. J. Computer Engineering and Design., 40(9): 27042711.

19. Bao Yanhong, $\mathrm{Xu}$ Taishan, Xu Lixiong. (2010) Cluster computing mode for transient stabilityconstrained preventive control implementation and total transfer capability calculation. J. Automation of Electric Power Systems., 34(1), 32-35.

20. Jain, T., Singh, S.N., Srivastava, S.C. (2008) Dynamic available transfer capability computation using a hybrid approach. J. IET Proceedings: Generation, Transmission \& Distribution., 2(6): 775-788.

21. Cui Kai, Fang, D.Z., Chung, T.S. (2003) Algorithm for analysis of power transmission limit under transient stability constraints. In: 2003 Sixth International Conference on Advances in Power System Control, Operation and Management APSCOM 2003. IET, Hong Kong, China. pp. 360365.

22. Wang Shengming, Xu Taishan, Xu Jianbing. (2011) Research and implementation of asynchronous concurrent scheduling model for online system. In: ICHCC High Performance Networking, Computing, and Communication Systems Second International Conference. Singapore. pp. 592-599. 